\title{
A DISPOSABLE CAPILLARY MICROPUMP USING FROZEN WATER AS SACRIFICIAL LAYER
}

\author{
Li-Wei Pan, Yu-Chuan Su, Sha Li and Liwei Lin \\ Berkeley Sensor and Actuator Center \\ Mechanical Engineering, University of California at Berkeley \\ Berkeley, CA 94720-2120
}

\begin{abstract}
A disposable capillary micropump has been successfully demonstrated by a one-mask microfabrication process using frozen water as the sacrificial layer. The micropump consists of two fluidic chambers (source and drain) and a $6.5 \mathrm{~mm}$-long, $5 \mu \mathrm{m}$-thick, naturally formed microchannel for capillary reaction. This prototype capillary micropump requires no electrical power and provides a maximum flow rate of $2 \mathrm{~nL} / \mathrm{min}$ with an operation volume of $10 \mathrm{~nL}$ in 30 minutes. The surface tension reaction force between the liquid sacrificial layer and the surrounding channel materials controls the heights of the microfluidic components such as chambers and channels naturally. Using water as the sacrificial layer has the advantage of simple and clean processing. As such, this self-primed process and the self-powered micropump may have great potentials in bio-medical applications, including disposable devices.
\end{abstract}

\section{INTRODUCTION}

It have been well documented that micropumps generally require high electrical power to operate and complicated manufacturing process to fabricate [1] Self-powered micropumps that are fabricated by very simple processing steps are attractive, especially, in the area of disposable applications. For example, emerging biological assays such as single molecule studies of DNA and cell adhesion analyses required a system that can provide nonpulsatile flows at extremely low velocity. Previously micropumps driven by the evaporation of water have been developed but the fabrication processes are complicated [2,3]. This work presents a new fabrication technology by using frozen water as a clean and easily removable sacrificial layer in the fabrication process for the

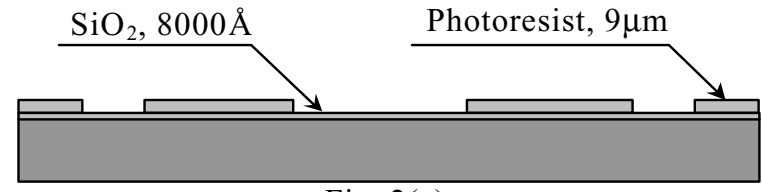

Fig. 2(a)

He-SF 6 Plasma, $100 \mathrm{~W}, 45$ seconds

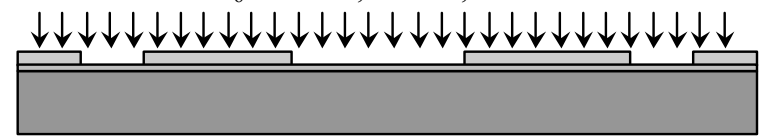

Fig. 2(b)

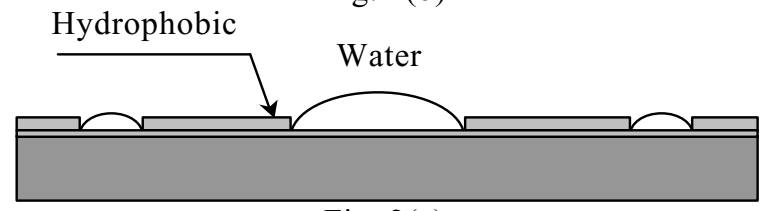

Fig. 2(c)

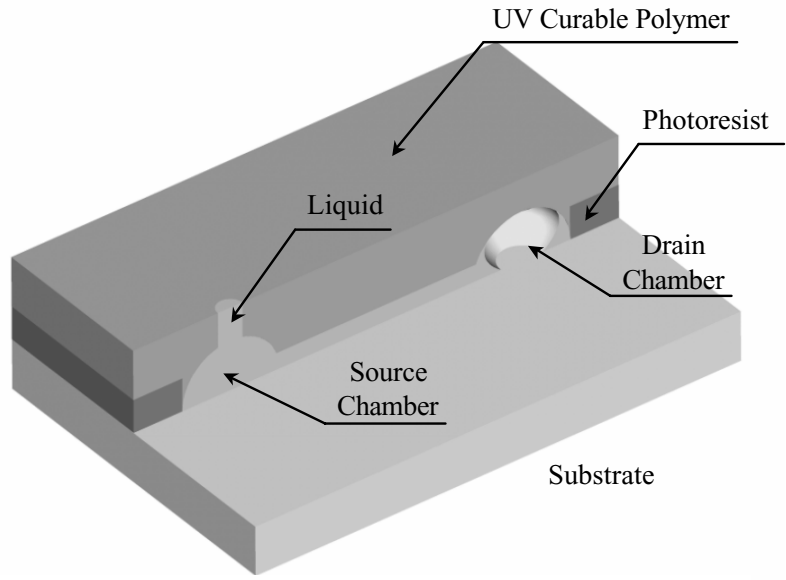

Fig. 1: The schematic drawing illustrating the working principle of the capillary micropump.

first time. The water sacrificial technique can provide smooth and continuous surfaces such that the dead volume can be reduced and the efficiency can be enhanced. This novel technology can also provide the encapsulation of liquid that has the potential for drug delivery applications.

\section{WORKING PRINCIPLE}

The working principle of the micropump is illustrated in Fig. 1. The whole device is composed of a source and a drain chamber and a long serpentine-shape microchannel. These components are fabricated on top of a hydrophilic surface. In the prototype demonstration, silicon wafer with a layer of thermal oxide has been used as the substrate. A photoresist layer is deposited and
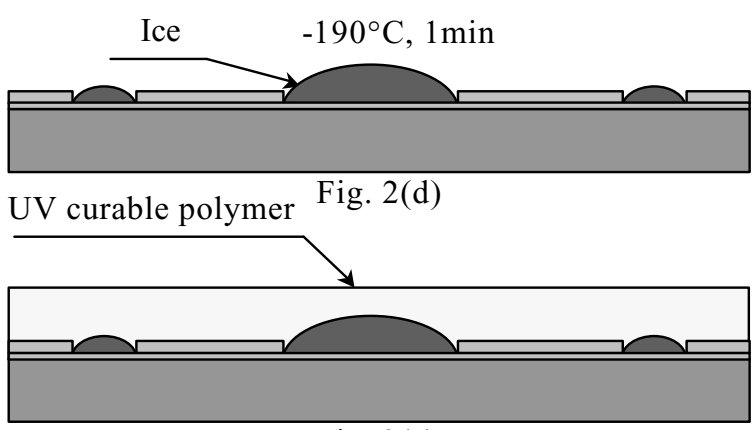

Fig. 2(e)

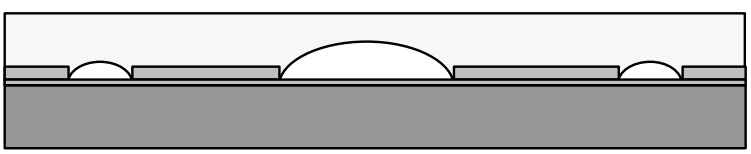

Fig. 2(f)

Fig.2: Fabrication sequence of the disposable capillary micropump using frozen water as the sacrificial layer 
patterned to define the chambers and channel areas as shown in Fig. 1 and UV-curable polymer is used to cover and seal the whole system. The critical process step is conducted by placing the sacrificial liquid onto the chamber and channel areas selectively utilizing the property of hydrophilic and hydrophobic surfaces for selective positioning. The sacrificial liquid is then frozen by lowering the substrate temperature to below the freezing temperature for the sacrificial liquid such that UV-curable polymer can be easily placed on top the solid surface. When the substrate temperature is increased, the frozen sacrificial solid becomes liquid again. The sacrificial liquid may evaporate completely to the environment or be kept inside the microstructures depending on the applications and the selection of polymers. In the micropump application as demonstrated in this work, water is used as the sacrificial layer and is driven out completely to complete the process either naturally or by placing the device into an oven to speed up the drying process.

Figure 2 shows the fabrication sequence of the prototype micropump. The process starts with growing a $0.8 \mu \mathrm{m}$-thick silicon dioxide on a clean silicon wafer. A $9 \mu \mathrm{m}$-thick photoresist (Shipley STR 1075) layer is patterned to define the microchannels and fluid reservoirs as shown in Fig. 2(a). A plasma process is applied to

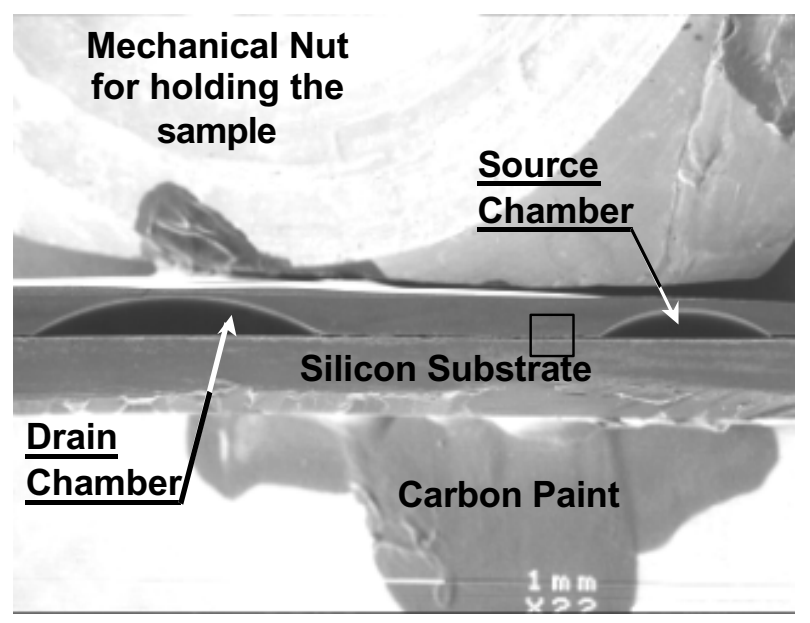

Fig. 3: The SEM microphoto showing the cross-sectional view of microchambers.

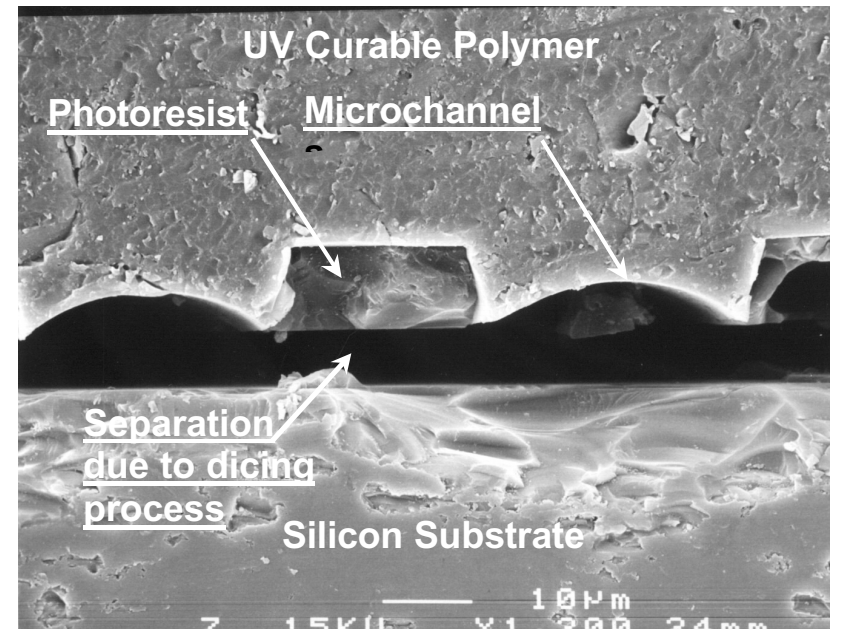

Fig. 4: The close view SEM microphoto of Fig. 2 showing the microchannels. make the surface of the photoresist hydrophobic [4] (Fig. 2(b)). Water is then poured onto the wafer and is automatically and selectively placed into the microchannels and reservoirs as shown in Fig. 2(c). The naturally formed water areas have different heights and shapes depending on the geometry of channels and the properties of hydrophobic/hydrophilic surfaces and working liquid. The substrate is then immersed into a liquid nitrogen bath for 1 minute and liquid water is transformed as solid ice as shown in Fig. 2(d). The ice serves as the sacrificial layer when a $250 \mu \mathrm{m}$-thick UV curable polymer is coated and cured as illustrated in Fig. 2(e). The water is driven out either naturally after a few days or by putting in an oven for a few hours to complete the process as shown in Fig. 2(f). Working liquid is supplied through a fluidic port opened at the source chamber. The hydrophilic surface inside the microchannel drags water automatically (self-prime) to the drain chamber by means of surface tension force as an operating capillary micropump.

Figure 3 is the cross sectional view of the fabricated micropump showing the source and drain chambers of $90 \mu \mathrm{m}$ and $204 \mu \mathrm{m}$ in height, respectively. The source chamber is defined by a square area of $600 \mu \mathrm{m}$ in width and the drain chamber is defined by a circular area of $1250 \mu \mathrm{m}$ in diameter. As a result, the surface

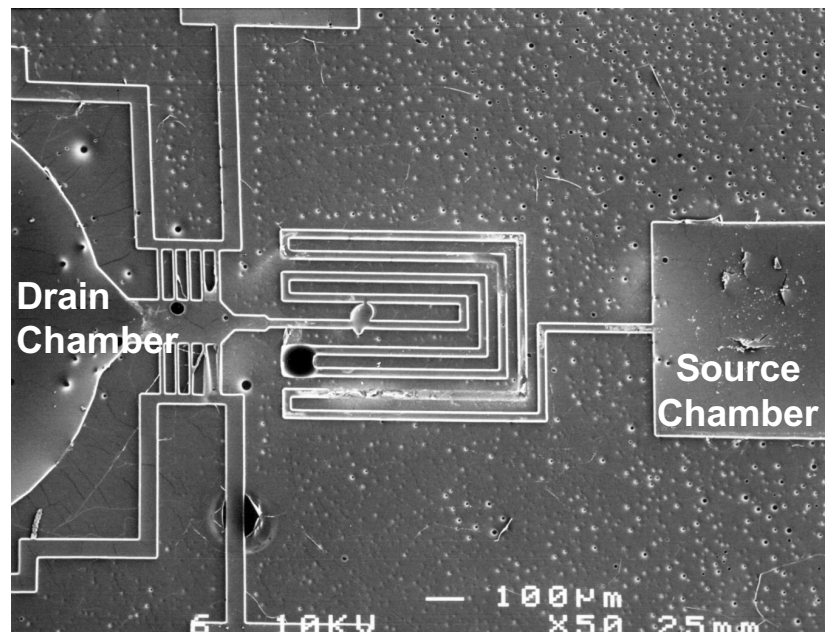

Fig. 5: The SEM microphoto showing the overview of the micropump.

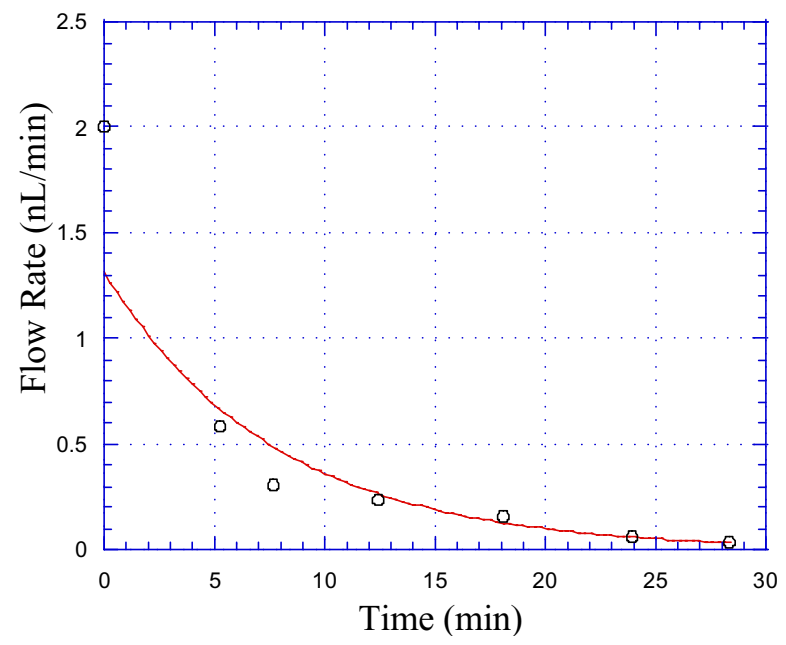

Fig. 6: Measured flow rate of the one-mask, disposable capillary micropump. 
tension force of the water droplets controls the height of these chambers naturally in the manufacturing process. The designated box area in Fig. 3 is enlarged and shown in Fig. 4, where the polymer layers (both photoresist and UV-curable polymer) are observed to be separated with the silicon substrate during the wafer dicing process. However, the shape of the two microchannels and the photoresist between them can be clearly observed. The sidewall of the microchannels seems to be hydrophobic that prevents penetration of the sacrificial water. The estimated height of the microchannels is $5 \mu \mathrm{m}$ while the width is $20 \mu \mathrm{m}$. Figure 5 shows the top SEM microphoto of the whole structure and the total length of the microchannel is $6.5 \mathrm{~mm}$ as shown. The micropump operates when a hole is poked open and liquid is poured into the source chamber. Capillary force drives the liquid toward the drain chamber at a high flow rate of about $2 \mathrm{nl} / \mathrm{min}$ initially.

Figure 6 is the measured flow rate of the micropump. It is suggested that flow rate decreases gradually until the capillary driving force is balanced by the air bubble pressure inside the drain chamber.

\section{DISCUSSIONS}

Using frozen water as sacrificial layer faces several key challenges. First, the process is sensitive to the conditions of environment such as temperature, humidity and airflow speed. In the prototype demonstration, none of these factors have been carefully controlled or maintained. The fabrication results are expected to improve if these factors can be controlled and optimized in the manufacturing environment. Temperature below freezing temperature $\left(0^{\circ} \mathrm{C}\right.$ for water) can keep the sacrificial liquid in the solid form for the next processing steps and liquid nitrogen appears to be an overkill that caused cracking of photoresist and polymer. Therefore, it is desirable that the process can be conducted at a temperature just below the freezing point without abrupt temperature changes in the process.

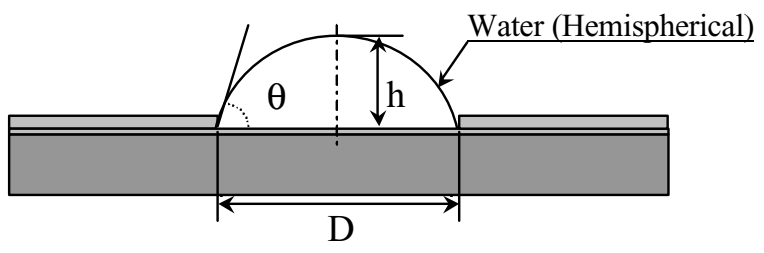

Fig. 7: The schematic drawing illustrates the model for microchamber and channel height design.

\section{$\underline{\text { Design rules }}$}

The naturally formed micro chambers and channels are decided by the balance of surface tension force. Because the maximum feature size in the prototype micropump is in millimeter scale, surface tension force is about at least 10 to 100 times larger than the gravitational force and water droplets can be regarded as hemispherical shapes as shown in Fig. 7 [5] and the height of the droplet, h, can be described as:

$$
h=D\left[\frac{1-\cos \theta}{2 \sin \theta}\right]
$$

where $\mathrm{D}$ is the characteristic length of the opening area (diameter for a circular opening area and width for a rectangular opening area) and $\theta$ is the contact of water to air and silicon dioxide interface. In this work, the heights of the source and drain chambers are calculated as $83 \mu \mathrm{m}$ and $173 \mu \mathrm{m}$ respectively and the height of the microchannel is calculated as $3 \mu \mathrm{m}$. These numbers are calculated based on the assumption that the contact angle of water and airsilicon dioxide interface is 31 degrees at room temperature. Since the micro chambers and channels are created during the transition of solid ice to liquid water and a new contact angle of water and UV curable polymer-silicon dioxide interface will be generated. Experimentally, the contact angle is measured by dicing the micro chamber right through the center as shown in Fig. 8 and the contact angle is measured as 43 degrees. Therefore, the heights of these microstructures are expected to be higher than the prediction using room temperature contact angle data. Based on Eq. 1, the calculated heights of the source and drain chamber used in this work are $118 \mu \mathrm{m}$ and $246 \mu \mathrm{m}$, respectively, and the height of the microchannel is calculated as $4 \mu \mathrm{m}$. These numbers are slightly higher than the experimental data.

The new contact angle may tend to increase the liquid volume in micro chambers and channels and may cause local distortions of the shapes of microstructures. The detail physical reaction requires further investigations. However, keeping the processing temperature just below frozen temperature during the curing process of UV-curable polymer may help alleviate this problem.

\section{Chamber/Channel Shapes and Surface}

The top UV curable polymer is detached from silicon substrate for surface analysis by etching away the photoresist layer in IPA (Isopropanol Alcohol). Figure 9 and 10 are SEM microphotos of microchambers and microchannels from the UV curable polymer surface. Cracks are observed on the surface due to abrupt processing temperature change of liquid nitrogen bath. Small cavities are also observed and they were formed due to the release of solvent gas from the photoresist and/or trapped and frozen water droplets from the environment after the liquid nitrogen bath [6]. These cracks and cavities may be eliminated or minimized by choosing appropriate photoresit together with optimized UV baking process and environmental control.

Figure 10 shows the close view of the marked box in Fig. 9 and two different shapes of microchannels (Type A and B) are identified. Type-A shape is formed when sacrificial water behaves like the schematic drawing in Fig. 2(e). Since the sacrificial water is confined at the intersection of the silicon dioxide layer and the sidewall of photoresist, a shallow microchannel shape is the result

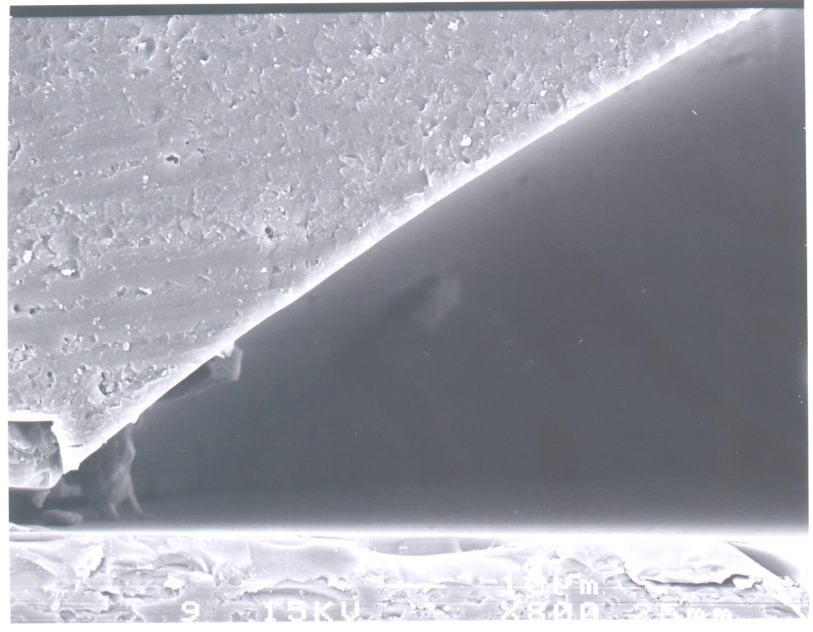

Fig. 8: The cross sectional microphoto showing the contact angle between water, UV curable polymer and silicon dioxide surface. 


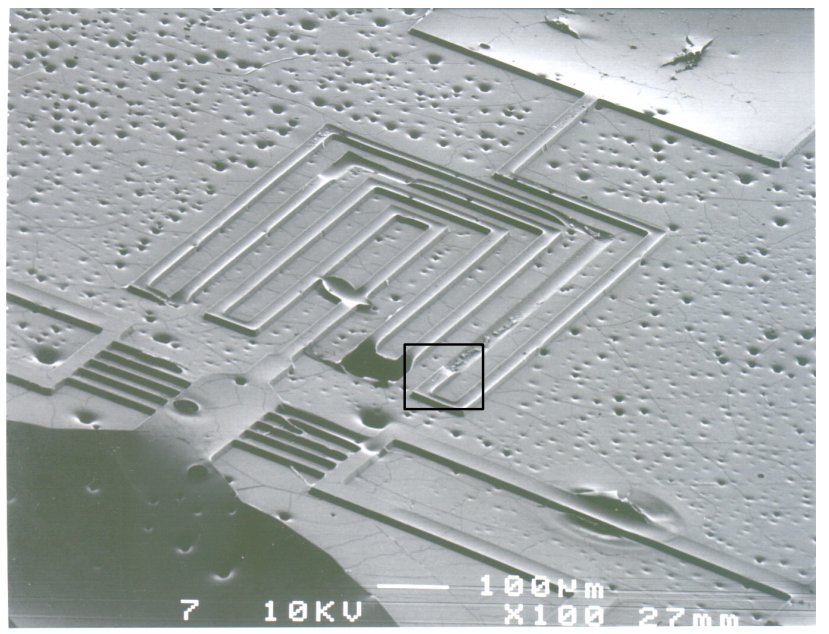

Fig. 9: SEM microphoto showing the microchannels formed by the ice sacrificial layer after removing silicon substrate.

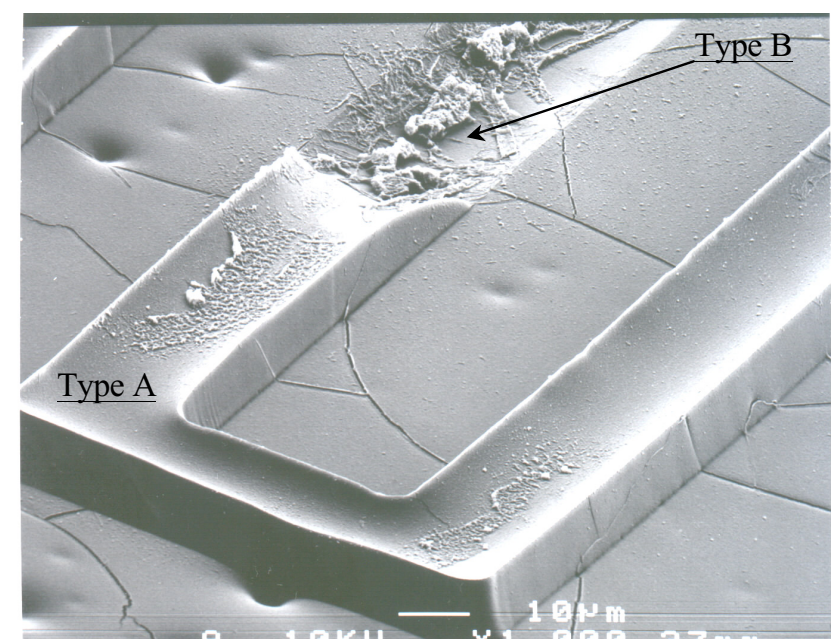

Fig. 10: The close view of the designated box area in Fig. 9.

and type-A shape is formed as shown in Fig. 10. On the other hand, it is observed that the sacrificial water can also fill either partially or completely the sidewalls of the microchannel such that the resulting microchannel shape can go from completely type-A to completely type-B as shown in Fig. 10. Type-B channel happens when the sacrificial water completely fills up the microchannel and the reverse of the channel shape after removing the photoresisit layer as shown in Fig. 10. Experimentally, a wide range of channel shapes from completely Type-A to completely Type-B has been observed in the same manufacturing process along a short piece of microchannel. The fundamental physics behind the various shapes of the microchannels require further investigations. However, it is believed that hydrophobic or hydrophilic surfaces of the photoresist sidewalls, the contact angle of wafer between different surfaces of photoresist, silicon dioxide and UV curable polymer at various temperature and the dynamic responses of the sacrificial water during the manufacturing process are key factors to be studied.

The internal surface of the microchannel formed by the water sacrificial layer is examined by SEM microphoto as shown in Fig. 10. Because there is no mechanical contact during the channel formation process, the surface is expected to be smooth as seen in the figure. Some particles are observed on the micro channel surface probably due to the contamination during the testing process. This smooth surface can minimize the resistance to the fluid flow.

\section{CONCLUSIONS}

A capillary micropump is fabricated utilizing frozen water as the sacrificial layer in the microfabrication process. This one-mask process is capable of making microfluidic components such as microchannels and reservoirs with variable heights as controlled by the contract angle of the sacrificial liquid and surrounding channel materials and the lithography-defined open areas. A prototype micropump driven by capillary forces is fabricated and tested as a demonstration vehicle. It has been measured that the micropump can provide a maximum flow rate of $2 \mathrm{~nL} / \mathrm{min}$ with an operation volume of $10 \mathrm{~nL}$ in 30 minutes. The heights of microchambers and microchannels can be designed by the contact angle of liquid and the size of opening areas. Controlled processing environment near the freezing temperature of sacrificial liquid is suggested to improve the yield of the process.

\section{ACKNOWLEDGEMENTS}

The authors would like to thank Mr. Ron Wilson for taking SEM pictures and Hyuck Choo and Jeonggi Seo of BSAC for valuable discussions. These devices were fabricated in the UC-Berkeley Microfabrication lab. This project is supported in part by a DARPA/MTO/BioFlips grant.

\section{REFERENCES}

1. M. Esashi, S. Shoji and A. Nakano, "Normally closed microvalve and micropump fabricated on a silicon wafe," Sensors and Actuators, vol.20, (no.1-2), p.163-9, 15 Nov. 1989.

2. V. Namasivayam, K. Handique, D.T. Burke, R.G. Larson, M.A. Burns, "Microfabricated valveless pump for delivering nonpulsatile flow," Proceedings of the SPIE- The international Society for Optical Engineering, vol. 4177, p.220-8, 2000.

3. N. Goedecke and A. Manz, "Towards Evaporation-Driven HPLC on a Chip: An Alternative Transport Process for Micro Analysis Systems," Proceedings of Micro Total Analysis Systems 2001, p. 375-6, 2001.

4. J. Seo and L.P. Lee, "Self-Assembly Templates by Selective Plasma Surface Modification of Micropatterned Photoresist," Proceedings, IEEE. Micro Electro Mechanical Systems, Las Vegas, U.S.A., p. 192-5, 2002.

5. A. Adamson, "Physical Chemistry of Surfaces", John Wiley \& Sons, $4^{\text {th }}$ edition, 1982.

6. C.S Hunag, B.Y Tsui, H.H Shieh, and R. Mohondro, "A novel UV baking process to improve DUV photoresist hardness.”,Proceedings of International Symposium on VLSI Technology Systems and Applications, Taipei, Taiwan, p.135-8., 1999. 